Transportation Research Forum

Specifying Automated Pavement Condition Surveys

Author(s): Jay K. Lindly, Frank Bell, and Sharif Ullah

Source: Journal of the Transportation Research Forum, Vol. 44, No. 3 (Fall 2005), pp. 19-32

Published by: Transportation Research Forum

Stable URL: http://www.trforum.org/journal

The Transportation Research Forum, founded in 1958, is an independent, nonprofit organization of transportation professionals who conduct, use, and benefit from research. Its purpose is to provide an impartial meeting ground for carriers, shippers, government officials, consultants, university researchers, suppliers, and others seeking exchange of information and ideas related to both passenger and freight transportation. More information on the Transportation Research Forum can be found on the Web at www.trforum.org. 


\section{Specifying Automated Pavement Condition Surveys}

Between 1994 and 2004, the number of U.S. and Canadian Departments of Transportation (DOTs) using automated techniques to record pavement surface distresses increased fourfold to approximately 30. Twenty more U.S. state agencies can be expected to automate techniques in the near future. The typical agency will use vans traveling at highway speeds to automatically measure roadway roughness, rutting, joint faulting, and cracking. This paper describes the upgrade of the Alabama Department of Transportation's automated pavement condition data survey specifications. The objective of the paper is to provide information concerning costs, standards, and survey methodology that will be valuable to other DOTs as they add automation to their systems.

\section{by Jay K. Lindly, Frank Bell, and Sharif Ullah}

\section{INTRODUCTION}

State Departments of Transportation (DOTs) face difficult highway maintenance and resurfacing decisions as funding becomes increasingly limited. A pavement management system (PMS) is a necessary tool to help decision-makers best preserve the condition of the road system.

The basis of any pavement management system is the condition survey. Many of these surveys have been conducted on foot or while driving slowly on the shoulder (windshield surveys). Inspectors write down or key in the types, amounts, and severities of surface distresses. That data is later combined with roughness data and translated into what is often called a pavement condition rating (PCR), usually on a 0-100 scale, where 100 represents a perfect pavement. Pavements with low PCRs are candidates for preventive maintenance, resurfacing, or reconstruction. These manual surveys and manual evaluation of data are becoming impractical for many larger agencies for at least three reasons:

- Safety: Manual raters are at risk simply by being on the pavement.

- Consistency: Manual rating is subjective, and there may be significant differences in the PCR ratings generated by different raters.

- $\quad$ Personnel/time: With downsizing, agencies may not wish to employ the staff to manually rate thousands of miles of roads. Unless a large staff is utilized, the relatively slower manual collection methods cannot be completed in a timely way.

Recent trends indicate that 20 or more DOTs will automate part or all of their pavement condition data collection/processing activities in the next few years. The objective of this paper is to describe recent Alabama Department of Transportation (ALDOT) work to incorporate automated pavement condition specifications of innovative highway agencies into its pavement management system. It reviews applicable standards, provides cost figures, and describes data collection and evaluation procedures that policy makers in other DOTs will find useful as they plan their conversion to automated procedures for PMS data collection.

\section{BACKGROUND}

Automated data collection surveys involve a van equipped with sensors and cameras traveling at highway speeds to detect the same types of pavement data that manual raters collect (Figure 1). Typically, laser sensors collect data on roughness as measured by the International Roughness Index (IRI), rutting, and joint faulting (see the Glossary for definitions of pavement condition data terminology). Downward-facing cameras provide images of surface distresses such as cracks. Data processing typically involves human interaction with surface 
Figure 1: Automated Pavement Condition Survey Vehicle

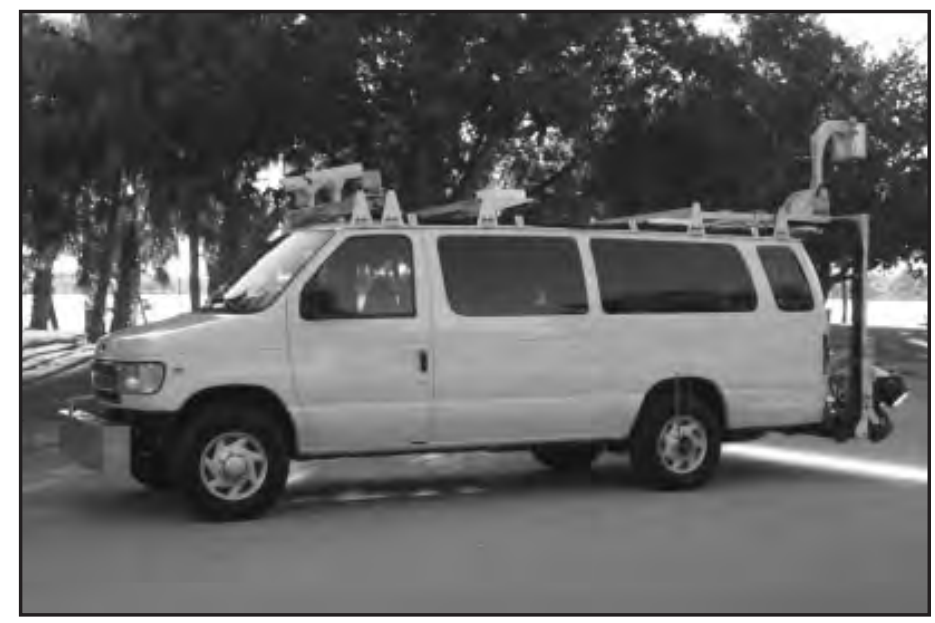

Source: International Cybernetics Corporation (2005)

distress images shown on computer monitors to identify and record the types and amounts of surface distresses.

\section{Current Literature}

Pavement condition survey methods are constantly improving. Pavement experts and software developers describe new systems where the computer analyzes the distress images without human aid, potentially saving labor costs and producing results almost in real time (Lee and Lee 2004, and Cheng and Glazier 2002). Camera technology is evolving, with line scan digital cameras beginning to replace the area scan digital cameras typically used to produce downward-facing images (Sokolic et al. 2004).

Traditionally, states have recorded different pavement distress types and even defined distresses differently. The American Association of StateHighway and Transportation Officials (AASHTO) recently issued a series of provisional standards to help standardize data collection. Researchers are evaluating the provisional standards to determine if they can be adopted without losing the usefulness of historical databases (Raman et al. 2004).

State DOTs are engaged in a variety of efforts to improve data acquisition and processing. The Pennsylvania and Florida DOTs have investigated areas such as quality assurance (QA) of the condition survey program and distress image quality and accuracy (Stoffels et al. 2003, and Gunaratne, Mraz, and Sokolic 2003).

\section{Recent Surveys}

Two recent studies reported results of questionnaires sent to pavement maintenance or pavement management engineers in U.S. and Canadian transportation agencies. Auburn University recently published results of a questionnaire returned by 27 of 46 DOT pavement maintenance engineers contacted (Timm and McQueen 2004). The study's objective was to gather information from states that have switched from manual to automated surveys and to provide information on current automated data collection practices. The study provides thumbnail sketches of the pavement distress collection practices of the 27 DOTs and summarizes those practices in a series of tables and graphs.

The National Cooperative Highway Research Program (NCHRP) published Synthesis 334, Automated Pavement Distress Collection Techniques (NCHRP 2004) that reported the results of 56 surveys returned by DOTs, the Federal Highway Administration (FHWA), and Canadian agencies. The NCHRP synthesis indicates that in 1994, only seven of 59 agencies surveyed used automated techniques to record pavement surface distresses. In 2004, the new survey indicated that 30 of 56 agencies 
collect data on at least some surface distresses automatically. The trend toward automation is clear.

Table 1 provides more detail concerning agencies' switch to automated systems (Timm and McQueen 2004, p. 77, and NCHRP 2004, p. 9). Almost all agencies collect roughness and rut data automatically, while a lower percentage of agencies collect joint faulting automatically.
$334,67 \%$ of responding agencies report doing so (NCHRP 2004, p. 9).

There are both staffing and technological reasons for the trends toward automation and the use of vendors. First, many agencies are not staffed sufficiently to provide one or more field crews to collect the data. Additionally, sensor and camera technologies are advancing rapidly, and many agencies wish to avoid the

Table 1: Automated Collection Survey Results

\begin{tabular}{lcc}
\hline \multirow{2}{*}{ Measurement } & \multicolumn{2}{c}{ Agencies Using Automated Collection (percent) } \\
\cline { 2 - 3 } & NCHRP Study & Auburn Study \\
\hline Roughness & 96 & 81 \\
Rutting & 91 & 81 \\
Joint Faulting & 59 & 41 \\
Distress Data & 54 & 56 \\
\hline
\end{tabular}

Sources: National Cooperative Highway Research Program (2004); Timm and McQueen (2004)

All three types of "sensor" data are processed automatically. A little more than half of the agencies collect distress images automatically. Of those agencies, only about half of them process the data automatically. Thus, though many agencies have switched to automation in the last decade, many others have yet to make the change.

\section{Transportation Agency Collection or Contract Collection}

Some agencies such as the Minnesota DOT collect and process their own pavement condition data (Mn/DOT 2003, p. 1). Other agencies such as the Oklahoma DOT pay a vendor to collect and process data (ODOT 2004, p. 2). Agencies such as the Indiana DOT collect data from only a few hundred feet of every road mile (Timm and McQueen 2004, p. 50), but most agencies collect automated data continuously in the outside lane of traffic (NCHRP 2004, p. 8). Agencies that collect distress data typically contract with a vendor to collect it. In the Auburn study, 56\% of responding agencies use vendors (Timm and McQueen 2004, p. 79); in NCHRP Synthesis need to continuously research and update this technology and instead rely on vendors to keep pace with advances. Finally, vendors with multiple clients can get economies of scale and lower costs than DOTs which only survey their networks periodically.

\section{Applicable Standards}

AASHTO has published provisional and full standards for use in automated pavement condition surveys (AASHTO 2004a and AASHTO 2004b):

- $\quad$ R 36-04, Standard Practice for Evaluating Faulting of Concrete Pavements

- PP 37-04, Standard Practice for Determination of International Roughness Index (IRI) to Quantify Roughness of Pavements

- PP 38-00 (2003), Standard Practice for Determining Maximum Rut Depth in Asphalt Pavements

- PP 44-01 (2003), Standard Practice for Quantifying Cracks in Asphalt Pavement Surface

AASHTO provisional standards do not become full standards until approved by two-thirds 
of the AASHTO member agencies, and the standards may well be changed significantly before approval.

Only PP 37-04 is currently used by a majority of agencies. PP 44-01 is used by several agencies for quantifying cracks, but so is the Distress Identification Manual for the LongTerm Pavement Performance Program (Federal Highway Administration 2003), a researchlevel tool that nonetheless has agency users. Many DOTs use their own agency-specific crack measurement standards. Likewise, a variety of standards (including the AASHTO provisional standards) are used by agencies that automatically collect rutting and joint faulting data (NCHRP 2004, p. 9).

\section{Suitability of Automated Results}

The results of statewide pavement condition surveys are typically used in pavement management systems that provide an overview of the agency's entire road network. These network-level surveys may provide data for several activities:

- Identifying pavement condition trends,

- Identifying candidate maintenance and resurfacing projects,

- Forecasting pavement performance, and

- Allocating funding

After candidate road sections for maintenance and resurfacing projects are identified, agency personnel visit those sections and perform a more detailed project-level survey, usually performed on foot. That survey first establishes or rejects the need for work and then identifies the controlling conditions present, which determines the maintenance or resurfacing method to be used, if any.

The manual surveys currently performed at the network level can approach or reach project level quality, including data on bleeding, patching, and identification of small cracks that automated surveys may miss. Some DOTs are reluctant to substitute automated surveys for manual surveys because they do not want to lose the manually collected data. If the manual data is not collected, the agencies must change their method for calculating PCR. However, a recent study confirms that automated surveys are appropriate for network-level coverage when quality levels are strictly monitored (Groeger et al. 2003, p. 116).

\section{ALDOT Background}

ALDOT administers approximately 11,000 centerline miles of highways, consisting of approximately $98 \%$ asphalt-surfaced roads and $2 \%$ concrete-surfaced roads. ALDOT began using manual pavement distress surveys in 1984, sampling 200 feet every mile and surveying its system once every two years. In 1996, it transitioned to automatic condition surveys performed by a vendor and discontinued manual condition surveys. In 2002, ALDOT started a QA program, manually rating 200 feet every 10 miles and comparing the manual results to the automatically-generated results. Significant discrepancies between ALDOT and vendor data occurred in areas such as distress types and extent of the pavement surface covered by those distresses. ALDOT also discovered significant differences between its linear referencing system (LRS) mileposts and the physical mileposts in the field, which made it more difficult to compare the same 200 foot sections.

Because of the results of the QA study and the outputs from its standard pavement management reports, ALDOT began to mistrust the system's results. The method of determining PCR had not been updated when manual surveys were replaced by automated surveys, even though human eyes and cameras "see" cracks differently (humans usually detect more cracks, which can lead to the automated systems' underreporting of low-severity cracking). During the metric system's brief reign, the system had been metricized and de-metricized, which introduced changes into the LRS that were not always captured by the vendor. Ultimately, ALDOT decided that it needed to overhaul its pavement management system to cope with these changes.

As described previously, most states follow practices for quantifying pavement cracks that are unique to that state. As a result of the investigations described in this paper, ALDOT decided to discontinue its unique practices for quantifying cracks and to begin following the new AASHTO PP 44-01. Under PP 44-01, 
some entirely different categories of cracks will be measured, and the amount of the cracking may be recorded in different units. Thus, while much of ALDOT's historic pavement condition data (particularly that taken by manual survey teams) is accurate, it is not directly comparable to the data that will be collected in the future, and its usefulness to the PMS will be reduced. However, the adoption of PP 44-01 by several states may eventually allow them to "pool” data for joint analysis.

\section{ALDOT INVESTIGATIONS}

ALDOT concluded that it must obtain more information about the needs of the users of its pavement condition data as well as determine the state-of-the-art of pavement condition data collection and processing before overhauling its PMS system. It sought input from the FHWA, sent a questionnaire to its own maintenance personnel, and consulted with other agencies.

\section{FHWA}

ALDOT and University of Alabama personnel met with a pavement management specialist from the FHWA Atlanta Resource Center and subsequently assembled a list of high-priority topics for investigation:

- Test frequency and lanes to be tested

- Conditions and distresses to be measured

- Standards to follow

- Crack severity level widths

- $\quad$ Area cracking (load associated and block) reporting parameter

- Reporting increments

- Standard wheelpath and lane dimensions

- QC/QA Program

\section{ALDOT Survey}

Ashort survey was distributed to themaintenance personnel in both the ALDOT central office and field offices, who are the primary end-users of ALDOT's pavement condition data. Thirtyseven of 56 individuals responded. Responses to three questions were particularly specific and provided information relevant to changing the pavement condition survey method:
- Maintenance personnel most desired two pieces of information from a pavement condition survey: PCR tabulated every mile (almost all responses) and average rut depth (three-quarters of responses). Other needs cited included IRI, amount of cracking, cross-slopes, date last resurfaced, and pavement buildup.

- The questionnaire asked respondents to list the crack width at the point it becomes significant to them (they usually ignore cracks less than this width when making maintenance decisions). Responses of 0.25 inches and 0.125 inches predominated. Network-survey-level digital cameras could detect cracks as small as 0.08 to 0.12 inch wide at the time of the questionnaire, which confirmed that automated condition surveys could detect cracks of importance to maintenance personnel. ${ }^{1}$

- $\quad$ Respondents also chose from a list of 12 distresses that they believe create a need for maintenance or resurfacing. Loadassociated cracking garnered the most responses, followed by rutting (nearly all respondents). Patching and potholes led the second tier of distresses.

\section{State Agency Contacts}

FHWA personnel provided sample condition survey requests for proposals and specification documents from eight states. Project team members read the documents and tabulated answers to its list of high-priority topics for investigation. Team members also conducted extensive telephone interviews with representatives from the DOTs of three states: Colorado, Louisiana, and Oklahoma. Pertinent results of these activities will be described in following sections.

\section{INVESTIGATION RESULTS}

The results of ALDOT's investigations allowed the project group to make decisions concerning the list of high-priority investigation topics it had compiled in consultation with the FHWA. Those decisions are described in the following paragraphs. 


\section{Test Frequency and Lanes to be Tested}

The Auburn study reports that 52\% of respondents collect condition data annually; $30 \%$ collect data biennially; 15\% collect interstate data annually and other road data biennially (Timm and McQueen 2004, p. 78). The project team recommended retention of the current ALDOT system of surveying National Highway System (NHS) roads annually and the remainder of its road system biennially. One of the uses of pavement condition data is to predict future PCR of roads to anticipate when they may be candidates for maintenance and resurfacing. In Alabama, resurfaced roads, particularly those off the NHS, typically last 10+ years before requiring significant maintenance activities, which will allow at least five data points over the life of the overlay if data is collected biennially.

Historically, ALDOT surveyed all highway types in both directions up to two lanes in each direction because ALDOT had considered doing differing resurfacing cycles for the truck lanes on certain routes. This practice continued with ALDOT's transition to automated data collection. The review of other transportation agencies' documents indicated that those agencies survey far fewer lanes. A simple change to surveying one lane in both directions on multi-lane highways and surveying one lane in the "primary" direction (north or east) for smaller facilities was selected and will significantly reduce ALDOT data collection costs.

ALDOT chose a data reporting increment of 0.01 mile or 52.8 feet. NCHRP Synthesis 334 reports that most agencies report data in increments ranging from 50 to 1,000 feet, and that many U.S. agencies use 0.1 mile (NCHRP 2004, p. 9). ALDOT's value is within the typical range reported, and values can be aggregated easily if longer reporting increments are desired.

\section{Conditions and Distresses to be Measured}

Prior to 2005, ALDOT collected the following highway condition data types:

- Load associated cracking

- Longitudinal cracking

- Transverse cracking

- Block cracking

- Patching

- Raveling

- $\quad$ Bleeding

- Rut depth

- Shoulder type and condition

- Coded remarks about various other parameters

The project team evaluated the arguments that limited distress types are all that are required for a network survey and found them compelling. In particular, AASHTO PP 44-01 simplifies crack quantification for asphalt pavements by limiting cracks to only those found in the

\section{Figure 2: ALDOT Wheelpath Definitions}

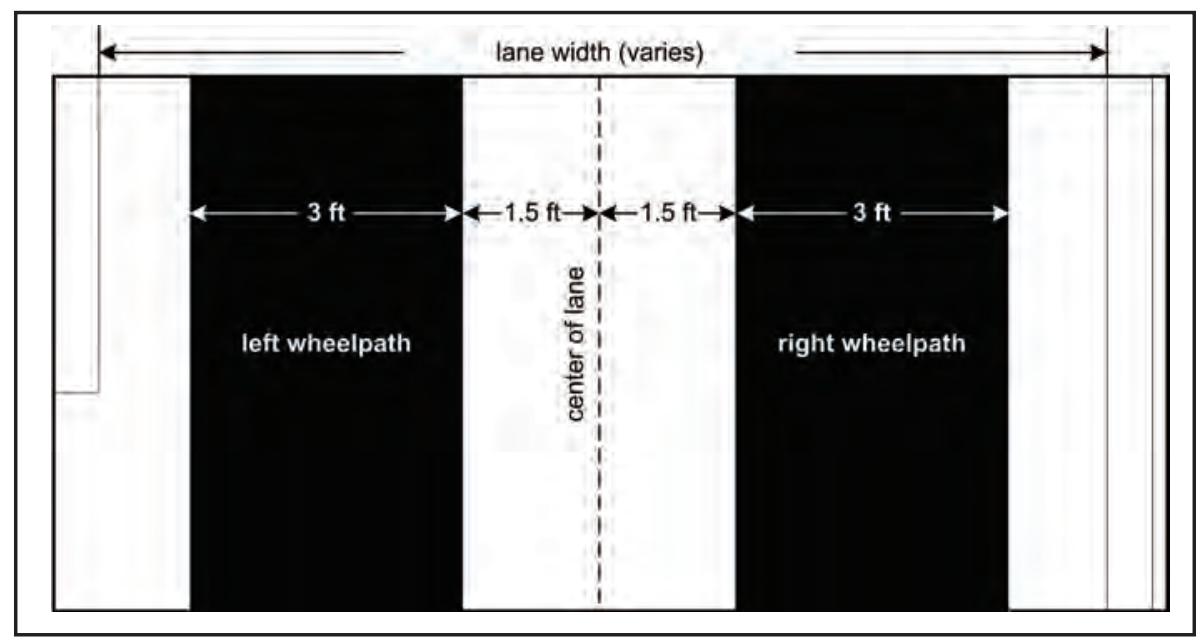


wheelpaths (load-associated cracks) and all others found outside the wheelpaths (non-loadassociated cracks). Figure 2 shows ALDOT's adaptation of the AASHTO P 44-01 diagram defining wheelpath and non-wheelpath areas.

In general, ALDOT adopted the AASHTO PP 44-01 designation for width of cracks as shown below:

- $\quad$ Severity level 1: Cracks having widths > $1 / 25$ inch and $\leq 1 / 8$ inch

- $\quad$ Severity level 2: Cracks having widths $>$ $1 / 8$ inch and $\leq 1 / 4$ inch

- $\quad$ Severity level 3: Cracks having widths $>$ $1 / 4$ inch

After reviewing other states' practices and considering the preferences expressed by ALDOT maintenance personnel, ALDOT selected the following condition data for evaluation in both flexible and rigid pavements (Table 2 lists accuracy and precision requirements for the data):

- IRI reported separately for the two wheel paths of the survey lane in inches/mile
- Transverse cracking reported in linear feet of cracking per 0.01 mile segment. To qualify, a single crack must be greater than six feet long and project within $30^{\circ}$ of perpendicular to the pavement centerline.

The following condition data was specified for flexible pavements:

- Load-associated cracking reports cracks in the wheelpaths that were not previously identified as transverse cracks. Loadassociated cracking is reported as the number of linear feet of road segment containing such cracking and cannot exceed 52.8 feet per 0.01 mile segment. When cracking occurs in both wheelpaths, the higher severity level of the two wheelpaths is reported.

- Non-load-associated cracking reports cracks longer than 1 inch (the minimum crack length as defined in PP 44-01) in the areas within the lane not identified as wheelpaths and were not previously identified as transverse cracks. Non-

Table 2: Accuracy and Precision Requirements for ALDOT Data Elements

\begin{tabular}{lcl}
\hline Data Element & $\begin{array}{c}\text { Required } \\
\text { Accuracy }\end{array}$ & \multicolumn{1}{c}{ Required Precision } \\
\hline $\begin{array}{l}\text { 1. Roughness (IRI) } \\
\text { 2. Cross slope, superelevation, } \\
\text { and grade data }\end{array}$ & $\pm 0.20 \%$ & $0.1 \%$ \\
3. Load-associated cracking & $\pm 10 \%$ & 0.1 linear foot per 0.01-mile \\
& & $\begin{array}{l}\text { sement } \\
\text { 4. Non-load associated cracking }\end{array}$ \\
& $\pm 10 \%$ & $\begin{array}{l}0.1 \text { linear foot per } 0.01-\text { mile } \\
\text { segment }\end{array}$ \\
5. Transverse cracking & $\pm 10 \%$ & 0.1 linear foot per 0.01-mile \\
6. Rut depth & \pm 0.1 inch & 0.1 inch \\
7. Raveling & Identical & present/not present \\
8. Patching & Identical & present/not present \\
9. Macrotexture & N/A & 0.01 inch \\
10. Joint faulting & \pm 0.1 inch & 0.1 inch \\
\hline
\end{tabular}

Accuracy is the required conformity to an ALDOT-measured value representing the "true" value. Precision is the exactness of the measured value, e.g., measured to the nearest 0.1 inch. 
load-associated cracks are reported as the number of linear feet of the 0.01 mile segment containing such cracking. The highest severity level present in the nonwheelpath areas is reported.

- Rutting reports mean and maximum values for both outside and inside wheelpaths for each 0.01 mile segment.

- Raveling reports instances where the aggregate and/or binder have worn away, coded as present or not present in each segment.

- $\quad$ Patching reports instances where patching exists and ride quality is affected, coded as present or not present in each segment.

- Macrotexture reports the mean and maximum values for wavelengths from 0.50 $\mathrm{mm}$ to $50 \mathrm{~mm}$ each 0.01 mile segment.

Though only about 2\% of ALDOT pavements have concrete surfaces, the following information was specified for rigid pavements:

- Transverse joint faulting reports mean and maximum values for each segment according to AASHTO R-36(04).

ALDOT's old distress collection procedure measured fatigue cracking by area and summarized most non-load-associated cracking by adding lengths of individual cracks. The new procedure measures the proportion of the longitudinal extent of the road that contains fatigue or non-load associated cracking. ALDOT's decision to parallel the requirements in PP 44-01 as much as possible motivated this change. In its data collection procedures, ALDOT also references PP 38-00 (2003) and R-36(04), and FHWA's Highway Performance Monitoring System Field Manual (FHWA 2002).

\section{Other Data}

Adding additional data to that already described results in relatively minor additional cost. For this reason, ALDOT decided to use the automated survey to collect data for other functions within ALDOT:

- Slope data including cross-slope of the pavement lane (percentage) and longitudinal grade (percentage). This is another example of sensor data, and accuracy and precision requirements are shown in Table 2.

- Global Positioning System (GPS) coordinates including longitude, latitude, elevation, and dilution of precision measurements. Positional accuracy for latitude and longitude must not exceed \pm 10 feet.

- Travel events such as pavement surface changes, railroad crossings, changes in number of lanes, and transitions from the lane specified for data collection. Event information is keyed in by personnel in the survey van.

For all cases involving measurements, these measurements are taken at the beginning of each 0.01 mile segment. In addition to extra data, digital right of way (ROW) images photographed with forward-facing cameras are specified for the beginning and midpoint of each segment, such that 10 -inch lettering is visible at a distance of 15 feet from the travel lane. These images are archived and can be used by central personnel to conduct preliminary inspections of sites without leaving the office.

Some vendors offer forward-facing cameras with the added capability of locating roadside objects in both the vertical and horizontal planes to within approximately 6 inches. Such images can be used to create accurate sign inventories or to monitor guardrail distance from the traveled way (Roadware 2005). ALDOT did not specify this service.

\section{DATA QUALITY}

ALDOT subscribes to the QA plan laid out in Section 5 of PP 44-01. The document describes qualification and training, equipment, validation sections, and additional checks that can be performed by the highway agency.

\section{Pre-Testing}

After experimenting with field verification of cracking data, ALDOT has adopted an approach similar to several of the states using automated collection of cracking data. Before statewide testing begins, ALDOT will select five to 10 , one-mile correlation sections that the vendor will rate using its equipment; ALDOT will then conduct a manual field rating of at 
least three randomly selected one-tenth-mile segments within them. Pavements displaying different distresses and levels of distress will be selected (most will be asphalt, given ALDOT's network). Vendor vans will then survey those sites. Vendor distress data and sensor data are expected to compare to ALDOT values within the accuracies specified in Table 2. ALDOT and the vendor will investigate differences between the datasets before statewide rating is allowed to proceed.

The QA procedure for sensor-collected data such as IRI, rutting, cross-slope, and texture uses a similar approach. ALDOT will select five to 102,000 -foot sections for the vendor to rate that may or may not correspond to the cracking sections. All of the sites will be rated at least five times, and the vendor results will be averaged and compared to departmentcollected data. ALDOT and the vendor will investigate differences between the datasets before statewide rating is allowed to proceed.

\section{Statewide Data Collection}

After the vendor has satisfied the requirement of the pre-testing, statewide data collection will begin. As a QA check for surface distresses, ALDOT will use the same software as the vendor to rate up to $5 \%$ of each set of data the vendor delivers to test for surface distress accuracy. ALDOT contract documents will specify that the vendor supply ALDOT with hardware, software, maintenance, and training to enable ALDOT personnel to perform these checks. Differences between the results obtained by ALDOT and the vendor will result in the vendor being required to re-rate some or all of the pavements rated in that set of data. The vendor should not have to recollect images to do the required re-processing.

ALDOT chose to follow the lead of Louisiana and Oklahoma in using a rolling approach to QA for data measured by sensors. Once per week, the consultant is required to return to a pavement section rated the previous week and resurvey that section. This data is compared with the prior week's data and must agree within the accuracies specified in Table 2. Also, the consultant is required to return to one of the initial correlation sites monthly to verify its results. If either comparison reveals errors, the data collected between visits is considered compromised and must be rerun.

Additionally, there are times when consultants may temporarily remove a test vehicle from ALDOT testing and times when the consultant may wish to operate multiple test vehicles in the state. ALDOT specified that each time a test vehicle enters the state, it must rate the sensor and condition data correlation sites, and the measurements must be correlated to produce sensor measurement differences of $5 \%$ or less between the consultant's vehicles.

ALDOT's long-term goal is to incorporate statistically-based QA procedures for surface distress data collection and processing. However, until further basic research is performed regarding variable pavement conditions, the imaging process, and the data reduction process, ALDOT intends to follow general nationwide current QA practice.

\section{Post-Data Collection}

After statewide surveys and data are summarized in the desired increments, the data will be checked using a variety of methods, as in the incomplete list below:

- Are one or more types of data missing for a segment?

- Does data exceed limits, e.g., in Alabama, does fatigue cracking exceed 52.8 feet in any segment?

- Is data from this year's run significantly different from the preceding year's run?

- Is data from one side of a multi-lane road significantly different from data for the other side?

\section{INTEGRATION INTO THE PMS}

When ALDOT began work on its PMS in 1984, there was vast experience in the maintenance areas of the organization. For this reason, a Delphi study was used to match engineers' qualitative opinions with quantitative data; the original PCR equation was developed from this study. Today, ALDOT employs fewer experienced engineers, and they are less likely to be able to devote time to such an extensive study. ALDOT plans to report separate 
indices for load-associated cracking, non-loadassociated cracking, roughness, and rutting that are also combined into a single PCR for prioritization. The indices and the PCR will be developed using the first year of new data. For each type of data, maps can be drawn at statewide and division levels to provide ALDOT personnel more tools to make local and network maintenance decisions.

ALDOT has historically used ratings across years for individual pavement sections to predict the future condition of that particular pavement. This was accomplished using a logarithmically-transformed linear regression that simulated the performance of pavements over the overlay life. The significantly different automated data that will be collected, however, makes continued use of the old prediction equation impossible. ALDOT has thus chosen a "family" approach for use with the new data. Routes with similar traffic and loading characteristics will be grouped together and, based on only one or two years of "new" data, will provide the necessary background to predict the future condition of that family of pavements. Critics may argue that this procedure is unwise because it forsakes years of historical data. However, Oklahoma DOT has recently followed this course successfully, and, as described earlier, ALDOT's distrust of its recent historical pavement condition data makes the decision necessary.

\section{COST}

ALDOT compiled cost data for three methods of pavement condition data collection and analysis: (1) manual data collection and manual computer entry using ALDOT personnel, (2) automated collection by vendor and distress image processing by vendor, and (3) automated collection using DOT personnel and distress image processing by DOT personnel. Those costs and the major benefits/drawbacks of each method are discussed below.

ALDOT updated old records to estimate 2004 costs for manual collection and data entry by ALDOT personnel. Because crews capturing data manually do not measure roughness, the estimate contained a provision for a separate van to drive the road system and collect IRI data at highway speed. The combined cost was approximately \$38/lane-mile. A major benefit of manual collection is the lower cost compared to the alternatives. Manual data collection also approaches the detail of a project-level survey, though project-level quality is not needed for a network-level study. However, there are major drawbacks to manual data collection:

- Increased safety risk for the field crew, particularly given the increased traffic on today's highways.

- The ALDOT manual survey crew only surveyed the first 200 feet of every road mile, giving an incomplete picture of the roadways. (Complete surveying would have been cost prohibitive.)

- This method does not provide "extras" such as continuous images from forward facing cameras, GPS data, and continuous cross slope measurements.

Discussions with FHWA personnel and other state DOTs indicated that vendor charges for a basic package of sensor data collection and processing, digital images and digital image processing, plus images from forward facing cameras were approximately \$50/lanemile in 2004. ALDOT's own vendor costs in 2004 were approximately \$53/lane-mile. NCHRP Synthesis 334 reports similar figures but cautions that some states have experienced higher charges on interstate roads or in urban, high-traffic areas (NCHRP 2004, pp. 44-45).

Vendor collection does not allow the DOT to control all aspects of automated data collection because quality control is in the hands of the vendor. However, ALDOT found benefits to vendor collection as compared to automated surveys performed by ALDOT personnel:

- ALDOT would not be required to hire and train six to eight technicians to perform data collection and distress image analysis.

- The vendor - not ALDOT - will have the responsibility to operate and maintain the high-tech survey van and remain on the cutting edge of camera and sensor improvements.

ALDOT contacted five state DOTs to inquire about costs to collect pavement condition data with their own van(s) and process distress images with their own personnel. Three DOTs did not have readily-available data, but two had 
recently completed life-cycle cost analyses of the data collection and analysis process. One contact reported a cost of approximately \$55/ lane-mile, while the second contact reported \$47-\$57/lane-mile, depending on the service life of the data collection van. These costs are very close to the costs for employing a vendor to collect and process data, and the principle benefits and drawbacks of the two methods were outlined in the previous paragraph.

\section{ALDOT'S SELECTED METHOD}

ALDOT selected vendor collection and processing of pavement condition data. The principle reason for not returning to manual data collection was survey crew safety: ALDOT did not want to return manual survey crews to roads with ever-increasing traffic volumes. The principle reasons for choosing not to collect and analyze pavement condition data with ALDOT personnel were concerns about hiring and training staff and the requirements of maintaining and upgrading the computers, sensors, and digital cameras in the van.

\section{CONCLUSIONS AND RECOMMENDATIONS}

The pavement condition survey is a requirement for a roadway PMS. Recent trends indicate that as many as 20 additional U.S. states will automate part or all of their pavement condition data collection/analysis activities in the next few years. This paper has described applicable standards, pertinent syntheses and studies, typical system components, test frequency, accuracy and precision requirements, costs, and QA/QC procedures frequently used in pavement condition studies. The information was presented in the context of ALDOT's reorganization of its condition survey procedures and will be useful to other governmental units embarking on automated pavement condition data collection and processing.

\section{Recommendations}

The authors present the following recommendations for states considering switching to automated pavement condition collection or modifying existing automated procedures:

- Consider collecting and reporting data according to AASHTO PP 44-01. Most states collect their own unique set of distress types using their own measurement scales. As more states use PP 44-01, the resulting standardized data can be compared easily from one region to another or pooled to compare and contrast pavement condition around the nation.

- Consider adding low-cost, extra services that will benefit other areas of the DOT to the standard data collected by the van. For example, extra data resulting in a traffic sign inventory system may benefit maintenance personnel even though signs are not a direct concern of the group that collects the pavement condition data.

- The authors cannot recommend one condition data collection procedure that fits every state. However, DOTs can consider the following information when making their decision:

- ALDOT manual data collection and processing costs approximately \$38/ lane-mile. Automated data collection and analysis - whether performed by DOT forces or through vendor contract - costs approximately \$50-\$55/lanemile in most areas of the country.

- Manual data collection is becoming increasingly difficult to continue in some areas because of safety concerns for the field crews and desires by some DOTs to reduce personnel.

- DOTs may need to consider their agencies' stance regarding new personnel hiring, the responsibilities of maintaining and upgrading sophisticated test van equipment, and the level of quality control they wish to exert over data measurement when they are choosing between purchasing vendor services and performing automated data collection with their own personnel. 


\section{Glossary}

Bleeding-Bituminous material that has arisen to the surface of an asphalt pavement, causing a slick, black surface.

Block cracking — Cracks in asphalt pavements that occur in roughly rectangular shapes, usually one square foot or greater in size.

Cross slope-The slope of the road surface perpendicular to the direction of travel that drains water from the road.

Distress images-Digital photographs of road surface distresses.

Grade-The change in elevation per horizontal distance traveled, expressed in percent.

Joint faulting-Difference in elevation between the sides of a joint in a concrete pavement that causes a bumpy ride.

Load-associated cracking-Cracks in the wheelpaths, generally caused by repeated passages of heavy wheel loads.

Macrotexture- The texture of the road surface having to do with the particle size gradation of stones in asphalt pavements.

Longitudinal cracking-Cracks in the pavement surface in the direction of vehicle travel.

Non-load-associated cracking-Cracks outside the wheelpaths, generally attributed to environmental causes such as age-hardening of asphalt.

Raveling-The wearing away of the pavement surface caused by the dislodging of aggregate particles.

Roughness - Variations in the longitudinal profile of the pavement surface that decrease ride quality.

Rutting-Longitudinal surface depressions in the wheelpaths.

Superelevation-The banking of a road going around a curve.

Surface distresses - Cracks, patches, etc. that indicate decay in the functionality of the pavement surface.

Transverse cracking-Cracks in the pavement surface at right angles to the direction of vehicle travel.

QA, Quality Assurance-Program established by the Department of Transportation to monitor quality of delivered data.

QC, Quality Control—Vendor-established program to ensure data delivery quality. 


\section{Endnotes}

1. There are differences in the crack detection abilities of the systems sold and used by different vendors and DOTs. For example, one system is operated only at night at speeds under 25 miles per hour ( $\mathrm{mph}$ ) and uses artificial lights to illuminate the pavement to meet crack detection requirements. Another prominent vendor operates at $55 \mathrm{mph}$ in the daytime and uses artificial lights to ensure that cracks are visible and shadows are avoided.

\section{References}

American Association of State Highway and Transportation Officials. Standard Specifications for Transportation Materials and Methods of Sampling and Testing, $24^{\text {th }}$ Edition. Washington, D.C., 2004a.

American Association of State Highway and Transportation Officials. AASHTO Provisional Standards. Washington, D.C., 2004b.

Cheng, H.D. and C. Glazier. Automated Real-Time Pavement Crack Deflection/Classification System. NCHRP-IDEA Program Project Final Report. Washington, D.C.: Transportation Research Board, 2002.

Federal Highway Administration. Distress Identification Manual for the Long-Term Pavement Performance Program (Fourth Revised Edition). FHWA-RD-03-031. McLean, VA, 2003.

Federal Highway Administration. Highway Performance Monitoring System Field Manual. OMB No. 21250028. Washington, D.C., 2002.

Groeger, J.L., P. Stephanos, P. Dorsey, and M. Chapman. "Implementation of Automated Network-Level Crack Detection Processes in Maryland.” Transportation Research Record 1860. Washington, D.C.: Transportation Research Board (2003):109-116.

Gunratne, M., A. Mraz, and I. Sokolic. Study of the Feasibility of Video-Logging with Pavement Condition Evaluation. CEE/FDOT/168-LO. Tampa, FL: CEE Department, University of South Florida, 2003.

International Cybernetics Corporation. 2005. Digital Imaging Vehicle [online]. [Cited 27 April, 2005.] Available from the World Wide Web: (http://www.internationalcybernetics.com/ imagingvehicle htm)

Lee, B.J. and H.D. Lee. "Position-Invariant Neural Network for Digital Pavement Crack Analysis.” Computer-Aided Civil and Infrastructure Engineering Vol. 19, Issue 2. Malden, MA (March 2004): 105-118.

Minnesota Department of Transportation. 2003. An Overview of Mn/DOT's Pavement Condition Rating Procedures and Indices [online]. [Cited 21 January, 2005]. Available from the World Wide Web: (www mrr.dot.state.mn.us/pavement/PvmtMgmt/RatingOverview.pdf).

National Cooperative Highway Research Program. NCHRP Synthesis 334 Automated Pavement Distress Collection Techniques. Washington, D.C.: Transportation Research Board, 2004. 
Oklahoma Department of Transportation. ODOT Statewide Pavement Management Report.

Oklahoma City, OK: Planning and Research Division, Pavement Management Branch, 2004.

Sokolic, I., M. Gunaratne, A. Mraz, and A. Nazef. Evaluation of Pavement Distress Imaging Systems. Presented at the 83d Annual Meeting of the Transportation Research Board.

Washington, D.C.: Transportation Research Board, 2004.

Stoffels, S.M., V.P. Ganesan, D.A. Morian, and C.E. Antle. Quality Assurance Procedures for Videologging and Pavement Condition Data Collection. FHWA-PA-2002-009-97-04. Washington, D.C.: Federal Highway Administration, 2003.

Timm, David H. and Jason M. McQueen. A Study of Manual vs. Automated Pavement Condition Surveys. IR-04-01. Auburn, AL: Highway Research Center, Auburn University, 2004.

Raman, M., M. Hossain, R.W. Miller, G. Cumberledge, H. Lee, and K. Kang. “Assessment of Image-Based Data Collection and the AASHTO Provisional Standard for Cracking on AsphaltSurfaced Pavements.” Transportation Research Record 1889. Washington, D.C.: Transportation Research Board (2004): 116-125.

Roadware Group, Inc. 2005. Surveyor [online]. [Cited 27 April, 2005]. Available from the World Wide Web: (http://www roadware.com/surveyor.htm)

Jay Lindly completed his schooling at Purdue University in 1987. Since then, he has taught and researched at the University of Alabama Department of Civil \& Environmental Engineering. His research interests include pavement management, traffic safety, and the highway/utility interface.

Frank Bell received his master of science degree from Auburn University in 1998. His experience includes construction scheduling, construction claims, and pavement management. He is currently employed by the Alabama Department of Transportation's Bureau of Materials \& Tests.

Sharif Ullah is working as a transportation engineer in the Champaign County Regional Planning Commission, Urbana, IL. He has a bachelor's degree in civil engineering from the Bangladesh University of Engineering \& Technology, and a master's degree in civil engineering from the University of Alabama. He is a certified engineer-intern in Alabama. 